\title{
Acute hemodynamic effects of non-invasive ventilation in patients with obesity hypoventilation syndrome
}

\author{
Pobeha P, Paranicova I, Trojova I, Tkacova R, Joppa P \\ Department of Respiratory Medicine and Tuberculosis, Faculty of Medicine, P. J. Safarik University \\ and L. Pasteur University Hospital, Kosice, Slovakia. pavol.joppa@upjs.sk
}

\begin{abstract}
OBJECTIVE: To evaluate the acute effects of volume-targeted non-invasive ventilation (NIV) on hemodynamic parameters assessed by impedance cardiography in patients with obesity hypoventilation syndrome (OHS). BACKGROUND: Despite the well-described beneficial effects of NIV using volume-targeted pressure support ventilation modes on respiration in OHS patients, questions were raised about the impact of this treatment on the cardiovascular system.

METHODS: In 15 patients (10 men; mean age, 55.8 \pm 9.3 years) impedance cardiography recordings were taken at baseline, after 120 minutes while on NIV and 20 minutes after NIV termination. A repeated-measures analysis of variance was used for comparisons.

RESULTS: Compared to baseline, a reduction in heart rate (from $80 \pm 11$ to $73 \pm 10$ beats per min, $p<0.05$ ) was observed on NIV whereas the stroke volume and cardiac index remained stable throughout all three assessed intervals ( $p=0.347, p=0.344$; respectively). The pre-ejection period increased on NIV (from $113 \pm 16$ to $127 \pm 20 \mathrm{~ms}, \mathrm{p}<0.05)$, and the left ventricular ejection time increased after NIV termination compared to baseline (from $259 \pm 25$ to $269 \pm 25 \mathrm{~ms}, \mathrm{p}<0.05$ ).

CONCLUSION: Volume-targeted NIV may acutely improve systolic time intervals without any negative impact on the left ventricular function in OHS patients (Tab. 2, Ref. 17). Text in PDF www.elis.sk

KEY WORDS: obesity hypoventilation syndrome, obstructive sleep apnoea, hypercapnia, non-invasive ventilation, impedance cardiography, cardiac output.
\end{abstract}

\section{Introduction}

Non-invasive ventilation (NIV) is a standard treatment for chronic respiratory failure in patients with obesity hypoventilation syndrome (OHS) and comorbid obstructive sleep apnoea (OSA) (1). However, despite the well-described beneficial effects of NIV using volume-targeted pressure support ventilation modes on respiration in OHS patients $(2,3)$, questions were raised about the impact of this treatment on the cardiovascular system (4). In particular, NIV was shown to acutely reduce cardiac output and stroke volume in disorders other than $\operatorname{OHS}(5,6)$. Nevertheless, studies on hemodynamic effects of NIV in patients with OHS (7, 8) are scarce, although there is evidence suggesting that longterm NIV use may improve left ventricular diastolic dysfunction

Department of Respiratory Medicine and Tuberculosis, Faculty of Medicine, P. J. Safarik University and L. Pasteur University Hospital, Kosice, Slovakia

Address for correspondence: P. Joppa, MD, PhD, Department of Respiratory Medicine and Tuberculosis, P. J. Safarik University and L. Pasteur University Hospital, Rastislavova 43, SK-041 90 Kosice, Slovakia. Phone: +421.55.6152640, Fax: +421.55.6152664

Acknowledgments: This work was supported by the Agency for Research and Development of the Slovak Republic under contract no. APVV-160158, and by grant VEGA 1/0220/17 of the Ministry of Education of the Slovak Republic. in such patients (8). Therefore, our study aimed to evaluate the acute effects of volume-targeted NIV on hemodynamic parameters in patients with OHS using a non-invasive method of impedance cardiography.

\section{Material and methods}

Clinically stable patients with OHS referred for the initiation of long-term NIV were consecutively recruited between 2017 and 2018. Arterial blood gas analysis and diagnostic sleep study were performed before the initiation. The diagnosis of OHS was defined by the combination of obesity [body mass index (BMI) $>30 \mathrm{~kg} /$ $\mathrm{m}^{2}$ ], sleep-disordered breathing, and awake daytime hypercapnia [resting arterial partial pressure of $\mathrm{CO}_{2}\left(\mathrm{paCO}_{2}\right)$ greater than 6.0 $\mathrm{kPa}$ ], in the absence of an alternate explanation for hypoventilation (9). All the patients underwent a transthoracic echocardiography with the assessment of left ventricular systolic function; those with an ejection fraction lower than $45 \%$ were excluded. The study was approved by the L. Pasteur University Hospital Ethics Committee and all the patients signed a written informed consent.

Patients underwent a night with automated positive airway pressure (PAP) device (Prisma 20A, Löwenstein medical, Germany) to titrate the expiratory PAP. On the morning following the titration, NIV was initiated using the bi-level PAP device (Prisma 30ST, Löwenstein medical, Germany) with a volume-targeted 
spontaneous/timed ventilation mode using oro-nasal masks. Tidal volume was set to $8 \mathrm{ml} / \mathrm{kg}$ of ideal body weight (3). Hemodynamic changes were measured during two hours of initial NIV. Measurements were performed using beat-to-beat non-invasive monitoring with impedance cardiography (Niccomo, Medis, Germany) as previously described (10). In brief, impedance cardiography recordings were taken after 15 minutes at rest in a supine position suitable for NIV, and three 60-second intervals were marked during the recording - baseline (pre-NIV), during the stable phase of NIV after 120 minutes on NIV, and 20 minutes after NIV termination (post-NIV). Within the marked intervals, the following parameters were analysed: oxygen haemoglobin saturation with a pulse oximeter $\left(\mathrm{SpO}_{2}\right)$, heart rate, left ventricle cardiac output to body surface area - cardiac index (CI), stroke volume, pre-ejection period (PEP), left ventricular ejection time (LVET), systolic time ratio (STR), ejection time index (ETI) and ratio (ETR).

\section{Statistical analyses}

To compare the hemodynamic parameters recorded pre-NIV, on NIV, and post-NIV, a repeated measures analysis of variance (RM-ANOVA) was used. Holm-Sidak test was applied for posthoc pair-wise comparisons. The results are presented as the mean \pm standard deviation. A two-tailed $P$-value of $<0.05$ was considered statistically significant. Analyses were conducted using SPSS for Windows software (version 14.0).

\section{Results}

Fifteen patients with OHS (10 men) naive to NIV were enrolled. They were generally late-middle-aged (mean age, $55.8 \pm 9.3$ years) with the mean body mass index of $49.7 \pm 7.1 \mathrm{~kg} / \mathrm{m}^{2}$. Sleep study parameters and arterial blood gas values are displayed in Table 1. All the patients suffered from severe OSA and hypercapnic respiratory failure with a moderate-to-severe hypoxemia (Tab. 1). On NIV, the average attained tidal volume was $515.6 \pm 68.8 \mathrm{ml}$, whereas expiratory and inspiratory PAP was $13.3 \pm 2.9$ and $23 \pm 3.7$ $\mathrm{kPa}$ on average, respectively.

Two hours of NIV therapy resulted in significant increases in $\mathrm{SpO}_{2}$ from the baseline that were followed by restitution to preNIV values 20 minutes after NIV termination (Tab. 2). Assessment of hemodynamic effects (see Table 2) showed that compared to

Tab. 1. Sleep study parameters and awake arterial blood gas values in the study cohort.

\begin{tabular}{lc}
\hline Variable & \\
\hline ESS, points & $9.1 \pm 3.1$ \\
$\mathrm{AHI}($ episodes/hour) & $108.1 \pm 21.5$ \\
Mean $\mathrm{SpO}_{2}(\%)$ & $77.3 \pm 8.7$ \\
$\mathrm{CT} 90 \%(\%)$ & $77.7 \pm 29.2$ \\
$\mathrm{paCO}_{2}(\mathrm{kPa})$ & $7.5 \pm 0.7$ \\
$\mathrm{paO}_{2}(\mathrm{kPa})$ & $6.6 \pm 0.8$
\end{tabular}

Data are presented as the mean \pm SD. ESS - Epworth Sleepiness Scale; AHI - apnoeahypopnea index; $\mathrm{SpO}_{2}$ - pulse oximetry saturation; CT90\% - cumulative sleep time percentage spent with pulse oximetric saturation below $90 \%$; $\mathrm{paCO}_{2}-$ arterial partial pressure of carbon dioxide, $\mathrm{paO}_{2}-$ arterial partial pressure of oxygen
Tab. 2. Hemodynamic changes during 2-hour non-invasive ventilation protocol.

\begin{tabular}{lcccc}
\hline Variable & Pre-NIV & On NIV & Post-NIV & p \\
\hline $\mathrm{SpO}_{2}, \%$ & $84.5 \pm 6.7$ & $89.7 \pm 5.8^{\mathrm{a}}$ & $85.7 \pm 6.4$ & $<0.001$ \\
$\mathrm{HR}$, beats $/ \mathrm{min}^{2}$ & $80 \pm 11$ & $73 \pm 10^{\mathrm{a}}$ & $77 \pm 12$ & $<0.001$ \\
$\mathrm{CI}\left(1 / \mathrm{min} / \mathrm{m}^{2}\right)$ & $2.4 \pm 0.3$ & $2.3 \pm 0.4$ & $2.5 \pm 0.5$ & 0.347 \\
$\mathrm{SV}, \mathrm{ml}$ & $73 \pm 15$ & $79 \pm 26$ & $80 \pm 27$ & 0.344 \\
LVET, ms & $259 \pm 25$ & $261 \pm 21$ & $269 \pm 25^{\mathrm{a}}$ & 0.018 \\
PEP, ms & $113 \pm 16$ & $127 \pm 20^{\mathrm{a}}$ & $113 \pm 22$ & 0.002 \\
STR & $0.45 \pm 0.10$ & $0.49 \pm 0.10^{\mathrm{a}}$ & $0.42 \pm 0.10$ & 0.003 \\
ETI $(\mathrm{ms})$ & $372 \pm 17$ & $377 \pm 19$ & $392 \pm 15^{\mathrm{a}}$ & $<0.001$ \\
ETR $(\%)$ & $30.9 \pm 3.3$ & $31.4 \pm 3.8$ & $34.0 \pm 3.2^{\mathrm{a}}$ & $<0.001$ \\
\hline
\end{tabular}

Data are presented as the mean (SD). p values were calculated using Repeated Measures One-Way ANOVA. ${ }^{a} \mathrm{p}<0.05$ versus Pre-NIV. NIV - non-invasive ventilation; $\mathrm{SpO}_{2}$ - pulse oximetry saturation; $\mathrm{HR}$ - heart rate; $\mathrm{CI}$ - cardiac index; $\mathrm{SV}$ - stroke volume; LVET - left ventricular ejection time; PEP - pre-ejection period; STR systolic time (PEP/LVET) ratio; ETI - ejection time index; ETR - ejection time ratio

pre-NIV, NIV application resulted in reductions in heart rate $(\mathrm{p}<$ 0.05 ) with no significant changes in stroke volume and CI. Indeed, both stroke volume and CI remained relatively stable throughout all three assessed intervals ( $\mathrm{p}=0.347, \mathrm{p}=0.344$, respectively). Furthermore, significant increases in systolic time intervals PEP and STR were observed on NIV compared to baseline, with restoration after NIV termination ( $\mathrm{p}=0.002, \mathrm{p}=0.003$; respectively). Measurements of LVET, ETI, and ETR revealed that there were no changes during the NIV period, nevertheless, significant increases in all these variables occurred after NIV termination compared to baseline $(\mathrm{p}<0.05$ for all).

\section{Discussion}

This is the first prospective study assessing acute hemodynamic changes during initiation of NIV in a well-defined group of stable OHS patients with severe OSA using impedance cardiography and its results are intriguing: effective NIV was accompanied by temporary reductions of heart rate and improvements in systolic intervals, without any deterioration of left ventricular systolic function. Previous studies on the effects of NIV on left ventricular function in patients with respiratory failure in disorders other than OHS yielded inconsistent results: acute reductions of stroke volume and CI were observed in some $(5,6)$ but not in all the studies (11). In the present study, the observed decrease of HR did not result in unfavourable reductions in either CI or stroke volume. In a case report of a single OHS patient with severe OSA, cardiac output was preserved despite a decreased heart rate during sleep on NIV suggesting beneficial effects of NIV on stroke volume (12), in agreement with our observations.

Using volume-targeted bi-level pressure support mode as an effective method of ventilation in patients with $\operatorname{OHS}(2,3)$ is the strength of this study, in association with its prospective design and consistent use of a pre-defined protocol in all the patients. In addition, bi-level pressure support has been previously suggested as a treatment of the first choice for OSA with marked nocturnal hyposaturation (13) and the patients in the studied cohort had coexisting severe OSA with up to $77 \%$ of the cumulative sleep time spent with a saturation below $90 \%$ (Tab. 1). The rather small cohort 


\section{8-250}

of studied patients suggests that our results should be considered preliminary and further investigations are needed to confirm these findings. Furthermore, our study protocol was implemented during the daytime and, therefore, studies investigating heart function while on NIV during sleep are required to complement our observations. The use of impedance cardiography for hemodynamic measurements may represent another study limitation; this method was shown to potentially over- or underestimate the results during NIV compared to the thermo-dilution method (14). Nevertheless, impedance cardiography is an established non-invasive method to assess hemodynamic functions in both experimental (15) and clinical (16) studies, which is reliable when assessing trends (17). In conclusion, our pilot study suggests that volume-targeted NIV is a safe method that may acutely improve systolic time intervals while it does not negatively impact left ventricular function in OHS patients with comorbid OSA. Longitudinal observations with longer follow-up periods are needed to clarify the effects of regularly used nocturnal NIV on left ventricular systolic function in such patients.

\section{References}

1. Masa JF, Corral J, Alonso ML et al. Efficacy of different treatment alternatives for obesity hypoventilation syndrome. Pickwick study. Am J Respir Crit Care Med 2015; 192 (1): 86-95.

2. Storre JH, Seuthe B, Fiechter R et al. Average volume-assured pressure support in obesity hypoventilation: A randomized crossover trial. Chest 2006; 130 (3): 815-821.

3. Murphy PB, Davidson C, Hind MD et al. Volume targeted versus pressure support non-invasive ventilation in patients with super obesity and chronic respiratory failure: a randomized controlled trial. Thorax 2012; 67 (8): 727-734.

4. Duiverman ML, Arellano-Maric MP, Windisch W. Long-term noninvasive ventilation in patients with chronic hypercapnic respiratory failure: assisting the diaphragm, but threatening the heart? Curr Opin Pulm Med 2016; 22 (2): 130-137.

5. Ambrosino N, Nava S, Torbicki A et al. Haemodynamic effects of pressure support and PEEP ventilation by nasal route in patients with stable chronic obstructive pulmonary disease. Thorax 1993; 48 (5): 523-528.
6. Lukácsovits J, Carlucci A, Hill N et al. Physiological changes during low- and high-intensity noninvasive ventilation. Eur Respir J 2012; 39 (4): 869-875.

7. Castro-Añón O, Golpe R, Pérez-de-Llano LA et al. Haemodynamic effects of non-invasive ventilation in patients with obesity-hypoventilation syndrome. Respirology 2012; 17 (8): 1269-1274.

8. Masa JF, Mokhlesi B, Benítez I et al. Echocardiographic changes with positive airway pressure therapy in obesity hypoventilation syndrome. Long-term Pickwick randomized controlled clinical trial. Am J Respir Crit Care Med 2020; 201 (5): 586-597.

9. Mokhlesi B, Masa JF, Brozek JL et al. Evaluation and management of obesity hypoventilation syndrome. An official American Thoracic Society clinical practice guideline. Am J Respir Crit Care Med 2019; 200 (3): e6-e24.

10. Lorne E, Mahjoub Y, Diouf $M$ et al. Accuracy of impedance cardiography for evaluating trends in cardiac output: a comparison with oesophageal Doppler. Br J Anaesth 2014; 113 (4): 596-602.

11. Summers RL, Patch J, Kolb JC. Effect of the initiation of noninvasive bi-level positive airway pressure on haemodynamic stability. Eur J Emerg Med 2002; 9 (1): 37-41.

12. Yoshida M, Ando SI, Kadokami T et al. Instantaneous restoration of cardiac output by noninvasive positive pressure ventilation in a patient with obesity hypoventilation syndrome. J Cardiol Cases 2010; 3 (1): e40-e42.

13. Slouka D, Honnerova M, Hrabe V, Matas A. The prediction of treatment failure of the continuous positive airways pressure. Bratisl Med J 2014; 115 (11): 704-707.

14. Castor G, Molter G, Helms J, Niedermark I, Altmayer P. Determination of cardiac output during positive end-expiratory pressure - noninvasive electrical bioimpedance compared with standard thermodilution. Crit Care Med 1990; 18 (5): 544-546.

15. Turkseven CH, Pekoglu E, Buyukakilli B. Hemodynamic effects of epinephrine in rats: evaluation by impedance cardiography. Bratisl Med J 2017; 118 (10): 613-617.

16. Krohova J, Czippelova B, Turianikova Z, Lazarova Z, Tonhajzerova I, Javorka M. Preejection period as a sympathetic activity index: a role of confounding factors. Physiol Res 2017; 66 (Suppl 2): S265-S275.

17. Heinink TP, Lund JN, Williams JP. Accuracy of impedance cardiography for evaluating trends in cardiac output. Br J Anaesth 2015; 115 (2): 322-323. 